\title{
Granulocyte colony stimulating factor in COS-IUI cycles
}

\section{Pradnya Devdas Shetty ${ }^{1 *}$, Nikita Lad ${ }^{1}$, Pallavi Vishwekar ${ }^{2}$, Mamta Vijay Shivtare $^{1}$}

\author{
${ }^{1}$ Department of IVF, Dr. D Y Patil University, Mumbai, Maharashtra, India \\ ${ }^{2}$ Department of Obstetrics and Gynaecology, Dr. D Y Patil Medical College and Research Centre, Mumbai, \\ Maharashtra, India
}

Received: 20 February 2019

Accepted: 11 March 2019

\section{*Correspondence:}

Dr. Pradnya Devdas Shetty,

E-mail: prads.shetty@gmail.com

Copyright: (C) the author(s), publisher and licensee Medip Academy. This is an open-access article distributed under the terms of the Creative Commons Attribution Non-Commercial License, which permits unrestricted non-commercial use, distribution, and reproduction in any medium, provided the original work is properly cited.

\begin{abstract}
Background: An unresolved assisted reproductive technique problem is the unresponsive, thin endometrium. Approximately $0.6 \%-0.8 \%$ of patients do not reach the minimum thickness. Using endometrial co culture, G$\mathrm{CSF}>130 \mathrm{pg} / \mathrm{mL}$ was associated with significantly improved pregnancy rate in ART cycles. This is a retrospective study that included all unexplained infertility cycles with controlled ovulation stimulation -IUI protocols. Aim was to note the effects of G-CSF on thin endometrium and pregnancy rate in G-CSF administered COS-IUI cycles.

Methods: This study was done in the IVF department of Dr D Y Patil University, Navi Mumbai, India. Thin endometrium was defined as ET $<7 \mathrm{~mm}$ on transvaginal ultrasound. Clomiphene citrate was used for ovulation induction in strengths of $100 \mathrm{mg}$ or $50 \mathrm{mg}$ on day 2 of their cycle based on the antral follicle count. Trigger used was injection $10,000 \mu \mathrm{g}$ urinary hCG. On the same day when the trigger injection was given, 300 units G-CSF was instilled into the uterus. Post 36 hours IUI was done under aseptic precautions .After 16 days $\beta$-hCG levels were done to determine whether there is a pregnancy.

Results: In present study,200 COS-IUI cycles were analysed.50 cycles showed a thin endometrium and in them GCSF was used. The chemical pregnancy rates was $32 \%$, the intrauterine pregnancy rate was $28 \%$, ectopic pregnancy rate was $4 \%$.

Conclusions: Present study concluded that G-CSF increases ET significantly in COS-IUI cycles in the event of thin endometrium. In view of small cohort size further larger randomized controlled trials may be required to substantiate the above conclusions.
\end{abstract}

Keywords: COS-IUI, G-CSF, IUI protocols

\section{INTRODUCTION}

The definition of unexplained infertility will always be a moving target as more research and better tests reveal more underlying causes of infertility.

In the present Indian scenario with the majority of the population affording only basic infertility evaluation, it would be worth to designate infertility as unexplained when a couple has had regular unprotected intercourse for one year (or 6 months when the female partner is 35 years or more) and all the tests of basic infertility evaluation return within normal limits. These tests include proper analysis of semen, documentation of regular ovulation, documentation of a normal uterus with normal uterine cavity and patent tubes. ${ }^{1}$ Unexplained infertility diagnosis is made when a couple fails to conceive despite there being no obvious cause, even after subjecting the patient to a complete workup. The average incidence of unexplained infertility is around $10-15 \%$. 
In cases of unexplained infertility, the conventional approach starts with expectant management, moving to ovulation induction combined with intrauterine inseminations. ${ }^{1}$ Ovulation induction (OI) sets a goal to develop at least one follicle in an otherwise anovulatory cycle. Controlled ovarian stimulation (COS) involves recruiting more number of follicles. ${ }^{2}$ The commonest protocol used is 5 days of clomiphene citrate $(50-100 \mathrm{mg})$ from day 2 to day 6 of menstrual cycle. The target is to get a 1-3 follicles of size $18-20 \mathrm{~mm}$. The aim of controlled ovarian stimulation is to achieve a sufficient number of fertilizable oocytes of good quality. It also helps in preventing ovarian hyperstimulation syndrome (OHSS) and multiple pregnancy. In controlled ovarian stimulation cycles a normal responder may give 8-15 follicles.

Clomiphene citrate is a selective estrogen receptor modulator with a structure that allows it to bind to hypothalamic estrogen receptors, this interferes with estrogen receptor replenishment in the hypothalamus, resulting in increased pituitary release of FSH.it is the drug of choice for inducing ovulation in women with oligo-ovulation and anovulatory cycles.it has been reported that in such patients it induces ovulation by 70 $80 \%$.in combination with IUI it has been used with ovulating patients also. Clomiphene citrate is widely used for ovulation induction in present practice.

Intrauterine insemination (IUI) is reasonable first line therapy for patients with normal sperm morphology more than $4 \%$, provided other semen parameters are normal. Sperm count, motility and morphology are important factors for success. A positive correlation between pregnancy rate and total motile sperm count is seen. A significant rise in pregnancy rate is seen with post wash total motile sperms of more than five million per $\mathrm{ml}$. The end point of any ovulation induction is the time for triggering ovulation. The trigger for ovulation is given when the leading follicle is $18-20 \mathrm{~mm}$ in diameter. hCG is the most commonly used drug for inducing the final maturation of the follicle and ovulation. Urinary derived hCG in the dose of 5000 IU or 10,000 IU IM is given and IUI is done 34-36 hours after the trigger in case of single insemination.

To achieve an effective pregnancy outcome when the follicle reaches the size of $18-20 \mathrm{~mm}$, an endometrium thickness of $7 \mathrm{~mm}$ is warranted. An unresolved ART problem is the unresponsive, thin endometrium. Approximately $0.6 \%-0.8 \%$ of patients do not reach the minimum thickness. ${ }^{3}$ Ultrasound is used to study the echogenicity of the endometrium. Three echo patterns have been described:

- Hyperechoic endometrium seen in luteal phase,

- Triple line endometrium seen in late follicular phase,

- Hypoechoic endometrium in early follicular phase.

A triple line endometrium with thickness of more than 7$8 \mathrm{~mm}$ is best conducive for pregnancy.
Cytokines are increasingly recognized as potentially important local regulators of ovarian function. Granulocyte colony stimulating factor(G-CSF) belongs to family of haemopoietic growth factors and is produced primarily by haemopoietic cells, although several nonhaemopoietic cell types, such as osteoblast, smooth muscle, endothelial, epithelial cells and human ovary, human endometrium as well as reproductive tissue cells have also shown to produce G-CSF. Some authors have reported the serum G-CSF concentration significantly increases during the ovulatory phase compared with all other phases, suggesting that G-CSF may play an important role in ovulation. After ovulation, the endometrium acquires the ability to implant the developing embryo within a specific time-window, termed the receptive phase. It has been reported that some cytokines may have an important function in achieving or maintaining pregnancy and may be essential members of the 'implantation window', whereas others may be supportive and/or redundant during this phase of the menstrual cycle(4).Using endometrial coculture, G$\mathrm{CSF}>130 \mathrm{pg} / \mathrm{mL}$ was associated with significantly improved IVF pregnancy chance. ${ }^{5}$

Administration of G-CSF does not seem to affect embryonic chromosomal constitution and therefore seems safe. ${ }^{6}$ G-CSF thus demonstrates divergent roles in reproduction, having distinct effects on endometrium and implantation. A potentially growth expanding effect on endometrium may be suspected from its role in establishing early endometriotic lesions. ${ }^{7}$

Thus, in women presenting with unexplained infertility with a thin endometrium, granulocyte colony stimulating factor can be used to acquire a desirable reproductive outcome. The objective of this study was to note the effects of G-CSF on thin endometrium and the pregnancy rates in G-CSF administered COS-IUI cycles.

\section{METHODS}

It was a retrospective study done in IVF department of Dr DY Patil University, Navi Mumbai, that included all unexplained infertility cycles with controlled ovulation stimulation -IUI protocols where for a thin endometrium G-CSF was used.

\section{Inclusion criteria}

- Unexplained infertility

- Endometrial thickness less than $7 \mathrm{~mm}$

- COS-IUI cycles.

\section{Exclusion criteria}

- Any medical or surgical illness

- Known causes of infertility

- Abnormal semen parameters. 
All the patients had a written informed consent form in which the procedure of IUI was explained to them. Thin endometrium was defined as endometrial thickness less than $7 \mathrm{~mm}$ on transvaginal ultrasound. All the patients underwent a baseline transvaginal ultrasound to determine their antral follicle count on day 2 of their cycle. In case of a normal ultrasound findings, clomiphene citrate was used for ovulation induction in strengths of $100 \mathrm{mg}$ or $50 \mathrm{mg}$ on day 2 of their cycle based on the antral follicle count. No additional gonadotropins were used in rest of course of treatment. Arginine sachets were given on 6th hourly basis for a period of 3-5 days prior to ovulation trigger. Ovulation trigger used was injection $10,000 \mu \mathrm{g}$ urinary $\mathrm{hCG}$ in all patients. On the day of hCG injection, G-CSF was instilled 300 units into the fundus of the uterus using an intrauterine insemination catheter of $12 \mathrm{~cm}$ length. Post $36 \mathrm{hrs}$ IUI was done with minimum of 15 million motile sperms under aseptic precautions. The patients were reviewed after 16 days for $\beta$-hCG levels to determine whether there is a pregnancy.

\section{RESULTS}

In all,200 COS-IUI cycles were analysed.50 cycles showed a thin endometrium and in them 300 units G-CSF was used. Figure 1 shows age distribution of the patients included in this study. Out of the 50 patients, 10 patients were under the age of $28 \mathrm{yrs}, 10$ were between 28$32 \mathrm{yrs}, 16$ were between $32-35 y$ rs and 14 were more than $35 y$ rs. The mean age of patients included in this study was 29.5 years.

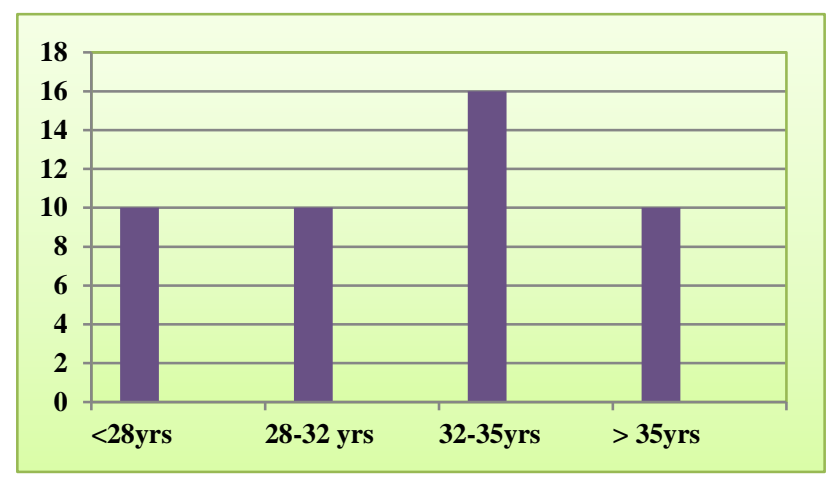

Figure 1: Age distribution of patients.

The patients received clomiphene citrate in the strength of $50 \mathrm{mg}$ or $100 \mathrm{mg}$ according to their antral follicle count. There was not much significant difference in antral follicle count in both the groups with respect of strength of clomiphene citrate used. Out of the 50 patients, 26 had received $50 \mathrm{mg}$ of clomiphene citrate and rest 24 received $100 \mathrm{mg}$ of clomiphene citrate. Figure 2 shows the distribution of clomiphene citrate among the patients.

When the endometrial thickness was compared before and after the instillation of G-CSF, the average increase in endometrial thickness after instillation of G-CSF was
$2.25 \mathrm{~mm}$. The lowest increase observed was $1 \mathrm{~mm}$ and the highest increase was of $3.9 \mathrm{~mm}$.

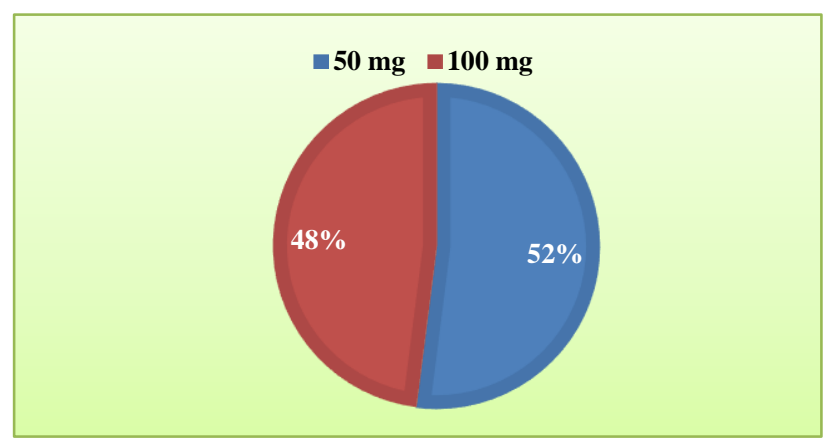

Figure 2: Distribution of clomiphene citrate.

Figure 3 demonstrates the effect of $50 \mathrm{mg}$ of clomiphene citrate, the average increase in endometrial thickness was $2.46 \mathrm{~mm}$. The lowest and highest increase in ET with $50 \mathrm{mg}$ of clomiphene citrate was $1 \mathrm{~mm}$ and $4.5 \mathrm{~mm}$ respectively.

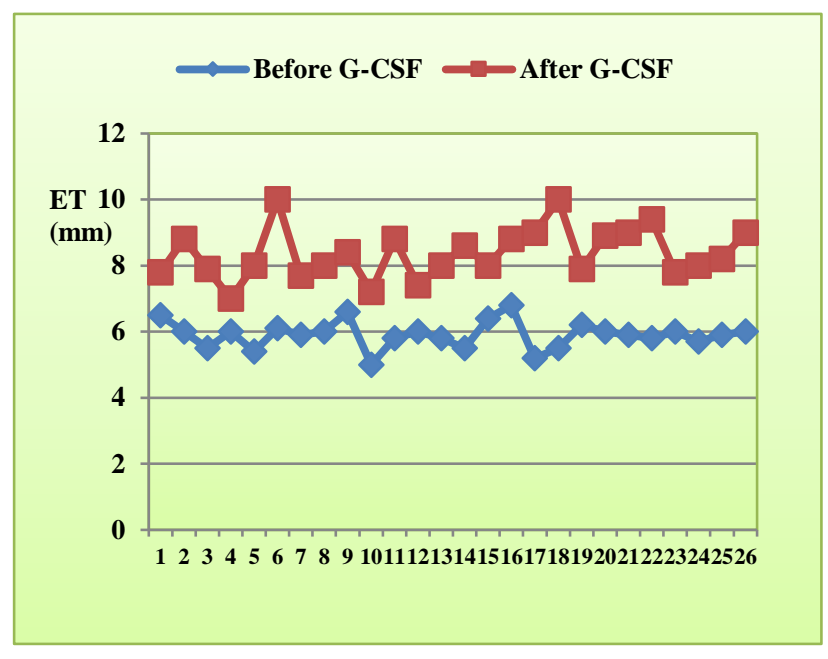

Figure 3: Endometrial thickness after infusion of GCSF among patients receiving 50mg clomiphene citrate.

Figure 4 demonstrates the effect of $100 \mathrm{mg}$ of clomiphene citrate, the average increase in endometrial thickness was found to be $2.06 \mathrm{~mm}$. The lowest and highest increase with $100 \mathrm{mg}$ of clomiphene citrate was $1 \mathrm{~mm}$ and $3.6 \mathrm{~mm}$ respectively. In the end, the effect with $50 \mathrm{mg}$ of clomiphene citrate was slightly better than $100 \mathrm{mg}$. There was a difference of $0.9 \mathrm{~mm}$ in the highest increase of the endometrial thickness between the two doses.

Finally, when the pregnancy rate was evaluated, there were 16 pregnancies with beta hCG levels above $30 \mathrm{mIU} / \mathrm{ml}$. Out of which 2 were ruptured ectopic pregnancies and 14 were intrauterine. When the intrauterine pregnancies were further followed up four of them continued till term and rest 10 were 1 st trimester miscarriages. 


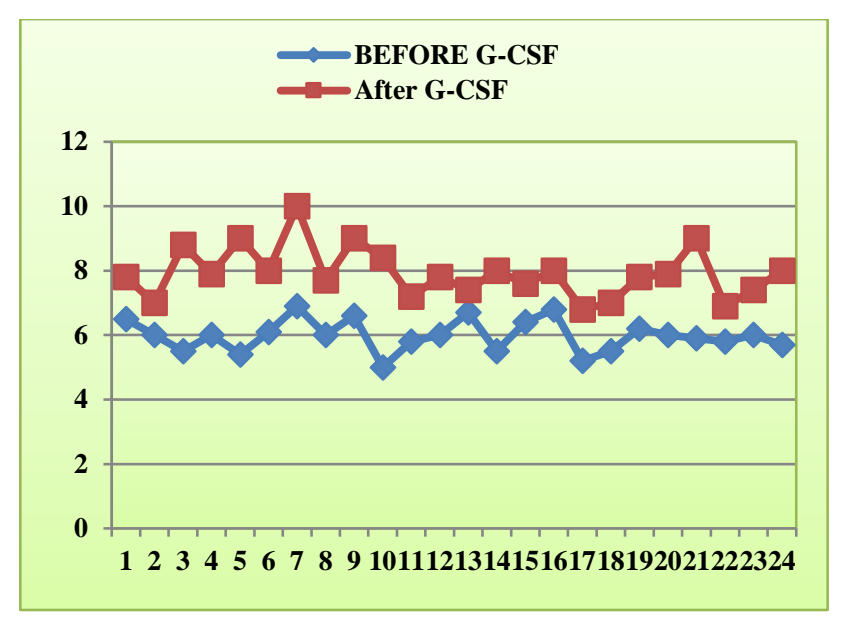

Figure 4: Endometrial thickness after infusion of GCSF among patients receiving $100 \mathrm{mg}$ clomiphene citrate.

\section{DISCUSSION}

There has been a widespread use of G-CSF in assisted reproductive techniques in recent years. Studies suggests effect of G-CSF in IVF cycles, but there are no such studies in COS-IUI cycles. Ours is the only study which quotes pregnancy outcome after use of G-CSF in patients with unresponsive endometrium in COS-IUI cycles.

Despite the small cohort size, our data are supportive of the idea that endometrial infusion with G-CSF may be effective in expanding chronically unresponsive thin endometrium. Authors reported 16 pregnancies in present study, out of which four continued till term. Large randomized controlled trials are necessary to further substantiate the effects of using G-CSF in IUI cycles as well for a better pregnancy rate. In a study done by Michal Kunicki et al titled "Evaluation of granulocyte colony stimulating factor effects on treatment-resistant thin endometrium in women undergoing in vitro fertilization".

They analyzed 37 women and found an increase in endometrial thickness as well as clinical pregnancy rate of around $18.9 \% .^{2}$ In a prospective observational cohort pilot study over 18 months done by N. Gleicher et al. titled, "A pilot cohort study of granulocyte colonystimulating factor in the treatment of unresponsive thin endometrium resistant to standard therapies". They analyzed 21 women and a $19.1 \%$ ongoing clinical pregnancy rate was observed, excluding one ectopic pregnancy. ${ }^{8}$ Since the cohort size of present study is not very large the results direct us to the discussion whether GCSF can be used in COS-IUI cycles too for increasing the endometrial thickness just like IVF cycles. Our results give a positive inclination towards the use of G-CSF in IUI cycles.

\section{CONCLUSION}

G-CSF increases the endometrial thickness significantly in COS-IUI cycles in the event of thin endometrium. In present study, chemical pregnancy rates was $32 \%$, the intrauterine pregnancy rate was $28 \%$, ectopic pregnancy rate was $4 \%$. In view of small cohort size further studies may be required to substantiate the above conclusions.

Funding: No funding sources

Conflict of interest: None declared

Ethical approval: The study was approved by the Institutional Ethics Committee

\section{REFERENCES}

1. Harry H. Infertility. Clinic Obstet Gynecol.54(4):642-3.

2. Kunicki M, Łukaszuk K, Woclawek-Potocka I, Liss J, Kulwikowska P, Szczyptańska J. Evaluation of granulocyte colony-stimulating factor effects on treatment-resistant thin endometrium in women undergoing in vitro fertilization. BioMed research international. 2014;2014.

3. Al-Ghamdi A, Coskun S, Al-Hassan S, Al-Rejjal R, Awartani K. The correlation between endometrial thickness and outcome of in vitro fertilization and embryo transfer (IVF-ET) outcome. Reproduct Biol Endocrinol. 2008;6(1):37.

4. Salmassi A, Schmutzler AG, Schaefer S, Koch K, Hedderich J, Jonat W et al. Is granulocyte colonystimulating factor level predictive for human IVF outcome?. Human Reproduction. 2005 May 12;20(9):2434-40.

5. Spandorfer SD, Barmat LI, Liu HC, Mele C, Veeck L, Rosenwaks Z. Granulocyte Macrophage-Colony Stimulating Factor Production by Autologous Endometrial Co-Culture Is Associated with Outcome for In Vitro Fertilization Patients with a History of Multiple Implantation Failures. Am J Reproduct Immunol. 1998;40(5):377-81.

6. Agerholm I, Loft A, Hald F, Lemmen JG, Munding B, Sørensen PD et al. Culture of human oocytes with granulocyte-macrophage colony-stimulating factor has no effect on embryonic chromosomal constitution. Reproductive biomedicine online. 2010;20(4):477-84.

7. Jensen JR, Witz CA, Schenken RS, Tekmal RR. A potential role for colony-stimulating factor 1 in the genesis of the early endometriotic lesion. Fertil Steril. 2010;93(1):251-6.

8. Gleicher N, Kim A, Michaeli T, Lee HJ, Shohat-Tal A, Lazzaroni E et al. A pilot cohort study of granulocyte colony-stimulating factor in the treatment of unresponsive thin endometrium resistant to standard therapies. Human Reprod. 2012;28(1):172-7.

Cite this article as: Shetty PD, Lad N, Vishwekar P, Shivtare MV. Granulocyte colony stimulating factor in COS-IUI cycles. Int J Reprod Contracept Obstet Gynecol 2019;8:1463-6. 